\title{
TEM study of Amorphous Phase Formation in Cobalt Nanoparticles
}

\author{
D. J. Sprouster ${ }^{1}$, D. J. Llewellyn ${ }^{1,4}$, P. Kluth ${ }^{1}$, L. L. Araujo ${ }^{1}$, J. D. Fitzgerald ${ }^{2}$, \\ B. Johannessen ${ }^{3}$ and M. C. Ridgway ${ }^{1}$ \\ ${ }^{1}$ Department of Electronic Materials Engineering, Research School of Physics and \\ Engineering, Australian National University, Canberra, ACT 0200, Australia \\ ${ }^{2}$ Research School of Earth Sciences, Australian National University, Canberra, 0200, \\ Australia \\ ${ }^{3}$ Australian Synchrotron Company Ltd, Clayton, Melbourne, VIC 3168, Australia \\ ${ }^{4}$ Australian National University Electron Microscopy Unit / Australian Microscopy and \\ Microanalysis Research Facility, Canberra, ACT 0200, Australia
}

Metallic nanometer-scale particles or nanoparticles (NPs) embedded in a dielectric matrix are of great scientific interest due to their novel size- and phase-dependent magnetic ${ }^{1}$, electronic ${ }^{2}$ and non-linear optical properties ${ }^{3}$, yielding novel applications in photonic ${ }^{4}$ and magnetic ${ }^{5}$ storage devices. For the most efficient integration of NPs in advanced devices, an ability to control the NP size, shape and crystallographic phase is essential. In bulk mono-elemental metals, recrystallisation typically inhibits formation of the amorphous phase subsequent to a quench from the melt or ion irradiation. In this work, cross-sectional transmission electron microscopy (XTEM) is used in conjunction with x-ray absorption spectroscopy (XAS) to structurally characterize the effects of ion irradiation on Co NPs embedded in a $\mathrm{SiO}_{2}$ matrix. As we demonstrate, an amorphous metallic phase can be achieved by ion irradiation of crystalline NPs embedded in an amorphous matrix, the latter potentially stabilizing the amorphous phase within a NP.

Crystalline Co NPs were synthesized in a $2 \mu \mathrm{m} \mathrm{SiO}_{2}$ layer grown on a $520 \mu \mathrm{m} \mathrm{Si} \mathrm{(100)}$ wafer by ion implantation and thermal annealing. The NPs were then irradiated with 9 $\mathrm{MeV} \mathrm{Au}$ ions to a fluence of $2 \times 10^{13} / \mathrm{cm}^{2}$ at room temperature (RT). To avoid thermally-induced recrystallization and/or diffusion and preserve the NP phase and size, XTEM samples were prepared entirely at RT or less. NP samples were initially bonded between two microscope glass slides using Loctite 363 (acetone resistant) and cured with UV light for $30 \mathrm{~min}$. The resulting sample stack was then ultrasonically cored on the cross-section using a $2.3 \mathrm{~mm}$ cutter then glued, using high-strength Araldite, into a $3 \mathrm{~mm}$ brass tube for support. The tube containing the sample cores was then cut into $400 \mu \mathrm{m}$ slices which were in turn glued to a Pyrex TEM stub using Superglue (soluble in acetone). After mechanical thinning, polishing and dimpling, the samples had a final centre thickness $<15 \mu \mathrm{m}$.

As a final step, the samples were milled in a GATAN 600 Duo Mill with $3.5 \mathrm{keV}$ Ar ions at $0.5 \mathrm{~mA}$ and an incident gun angle of $15^{\circ}$. The sample stage was in thermal contact with a liquid nitrogen reservoir maintaining a cold sample temperature to limit any ionbeam induced heating. Once a perforation appeared at the $\mathrm{SiO}_{2} /$ glass interface, the ion gun angles were reduce to $9^{\circ}$ and milled for a further $30 \mathrm{~min}$ to achieve the optimal layer thickness and maximum thin area for microscopy measurements. Samples were then examined with a Philips CM 300 microscope operating at $300 \mathrm{kV}$. XAS samples were prepared following the method described in ${ }^{6}$. 
Figure 1 shows XTEM micrographs of the embedded NPs before (a) and after (b) irradiation. The NPs are initially $\sim 3.3 \mathrm{~nm}$ in diameter and grow slightly, to $\sim 4 \mathrm{~nm}$, after irradiation. Size distributions determined from XTEM and small-angle X-ray scattering yield comparable results. Electron diffraction demonstrates the well-defined and continuous rings resulting from randomly-oriented polycrystalline Co NPs vanish upon irradiation, consistent with the formation of an amorphous phase. The XAS results shown in Figure 2 confirm the short-range atomic structure of the NPs changes significantly upon irradiation, with an increase in structural disorder, decrease in coordination number and complete absence of scattering from beyond the first coordination shell, again entirely consistent with the formation of an amorphous phase within the embedded Co NPs.

\section{References}

[1] F. Luis et. at., Phys. Rev. B. 65, 094409 (2002).

[2] J. Hormes et al., J. Appl. Phys. 97, 10R102 (2005).

[3] E. Cattaruzza et al., Appl. Phys. Lett. 73, 1176 (1998).

[4] E. Cattaruzza et. al., Phil. Mag. B 82, 735 (2002).

[5] V. Beyer et al., J. Appl. Phys. 104, 024512 (2008).

[6] D. Sprouster et al., submitted to Phys. Rev. B (2009).
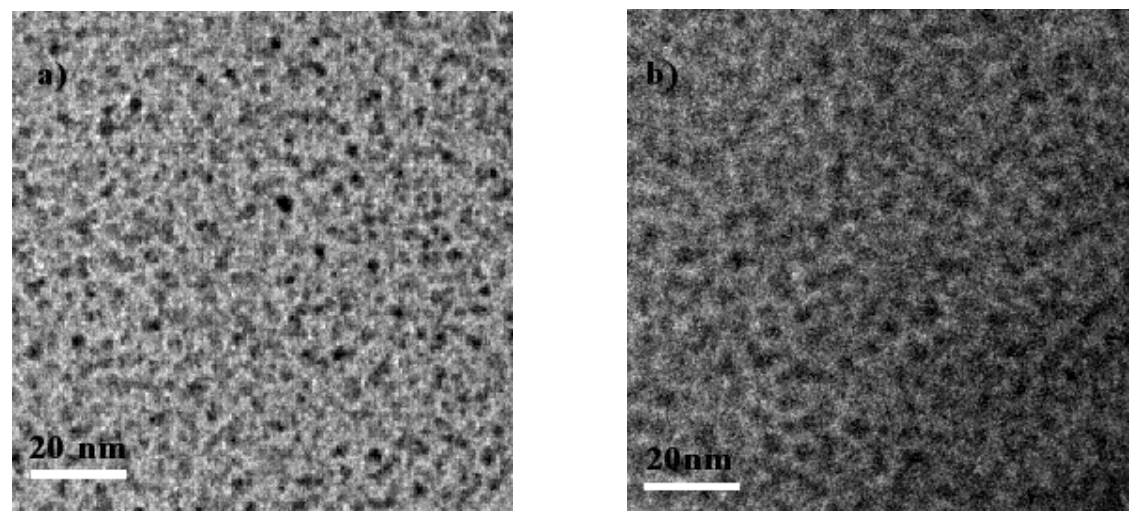

Figure 1. XTEM micrographs of (a) unirradiated and (b) irradiated NPs

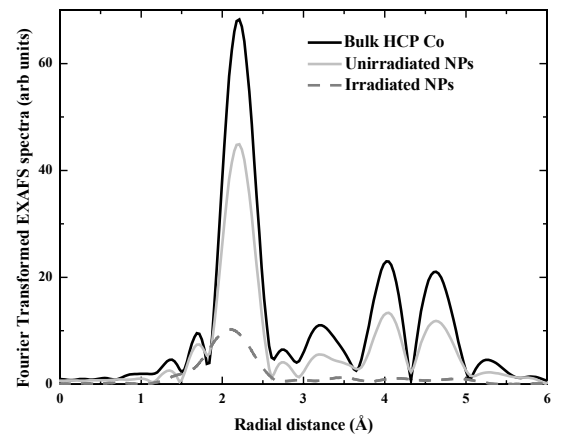

Figure 2. Fourier transformed EXAFS spectra for bulk HCP Co and Co NPs before and after irradiation.

*Email:djs109@rsphysse.anu.edu.au 